\title{
Management of hypertension in the elderly: Looking for a trade-off between cardiovascular prevention and serious adverse events
}

\author{
Agata Buonacera, Benedetta Stancanelli, Lorenzo Malatino \\ Academic Unit of Internal Medicine and Hypertension Centre, Department of Clinical and Experimental \\ Medicine, University of Catania, c/o Cannizzaro Hospital, Catania, Italy
}

\begin{abstract}
Despite the effectiveness of anti-hypertensive therapy is well recognized, the most adequate target to achieve in the management of hypertension in the elderly is still under debate. Indeed, looking at the balance between benefits and risks, accumulating evidence has recently specifically addressed this issue, focusing on controversies. Evidence surrounding a more aggressive treatment may lead to a turnaround in the clinical care of older adult hypertensives, but a balanced trade-off between benefits and risks needs to be programmed and achieved, particularly in this clinical setting. We argue whether and how the results from recent trials could influence the management of hypertension in older people.
\end{abstract}

\section{Introduction}

Blood pressure (BP) increases with age and so influences the relative cardiovascular (CV) risk and mortality from CV causes [1]. Therefore, BP management has become relevant in the clinical care of older adults. Older hypertensives, however, belong to a largely heterogeneous

Corresponding author: Prof. Lorenzo Malatino, Internal Medicine, University of Catania, c/o Cannizzaro Hospital, Via Messina 829, 95126 Catania, Italy. Tel. +39.095 .7262582 - Fax +39.095 .7262053 .

E-mail:malatino@unict.it

Key words: Arterial hypertension; antihypertensive therapy; elderly; blood pressure target.

Contributions: AB, BS, manuscript drafting; LM, manuscript revision for its intellectual content.

Conflict of interest: The Authors declare that they have no conflict of interest.

Received for publication: 24 April 2018

Accepted for publication: 11 May 2018

(C) Copyright A. Buonacera et al., 2018

Tipografia PI-ME Editrice, Italy

Monaldi Archives for Chest Disease 2018; 88:960

doi: 10.4081/monaldi.2018.960

This article is distributed under the terms of the Creative Commons Attribution Noncommercial License (by-nc 4.0) which permits any noncommercial use, distribution, and reproduction in any medium, provided the original author(s) and source are credited. population, because advanced age is characterized by comorbidities, frailty, deterioration in cognitive status and lack of autonomy in daily activities, all contributing to the complexity of the older patient's care. Furthermore, pathophysiological changes in baroreflex activity, cerebral autoregulation and arterial stiffness are usually observed in elderly patients, particularly in presence of long-standing hypertension history and/or isolated systolic hypertension (ISH). For all these reasons, BP management in elderly hypertensives still remains a challenge.

\section{Current state of the art - Hypertension studies}

The Hypertension in the Very Elderly Trial (HYVET) is the landmark study in the management of arterial hypertension in the elderly, giving ground to the current guidelines on this issue. The HYVET first demonstrated that BP reduction in elderly hypertensives is beneficial, even in patients aged $\geq 80$ years, in whom benefits were related to a target of systolic BP (SBP) $<150 / 80 \mathrm{mmHg}$ [2]. Indeed, the reduction in mortality from any cause $(-21 \%, \mathrm{P}=0.02)$, fatal stroke $(-39 \%$, $\mathrm{P}=0.05)$ and heart failure $(-64 \%, \mathrm{P}<0.001)$ with active antihypertensive treatment resulted impressive. HYVET thus gives strong evidence of benefit in reducing BP even in octogenarians. Of note, HYVET excluded from the recruitment ill and frail octogenarians (i.e. patients with dementia, accelerated hypertension, secondary hypertension, haemorrhagic stroke in the 6 months before recruitment, symptomatic heart failure, and so on).

On the other hand, many observational studies found that in elderly patients, especially in the frailest ones, the lower are BP values the greater is the mortality rate. In this respect, a recent cohort study observed a terminal decline of $\mathrm{BP}$ in octogenarians regardless their frailty status, so suggesting that it can be accounted for by their close proximity to death and not by BP reduction strategy on itself [3]. Although this finding needs further investigation, it seems to apparently reassure clinicians about the feasibility of an appropriate BP reduction in older hypertensives.

As to the baseline BP needing to start antihypertensive medication in elderly patients ( $\geq 80$ years), the European Society of Hypertension (ESH) and European Society of Cardiology (ESC) Guidelines for the management of arterial hypertension [4] identified a threshold of SBP $\geq 160 \mathrm{mmHg}$ (Class I; Level of Evidence A). Moreover, ESH/ESC guidelines [4] recommended a SBP goal of $140-150 \mathrm{mmHg}$ in people $<80$ yr-old (Class I; Level of Evidence A) and fit octogenarians (Class I; Level of Evidence B). A tighter BP goal may be considered for healthy hypertensives < 80 yr-old (Class IIb; Level of Evidence C), starting antihypertensive medication for a baseline SBP range between 140 and $159 \mathrm{mmHg}$. BP target in the management of frailest hypertensives should be tailored on the patient (Class IIb; Level of Evidence C). Therefore, in the last decades there was no shared opinion about the optimal and unique BP target to achieve in the management of arterial hypertension in the elderly. 
Thus, the Systolic Blood Pressure Intervention Trial (SPRINT) [5], showed for the first time that in non-diabetic hypertensives older than 50 years with increased CV risk, an intensive BP control (SBP target $<120 \mathrm{mmHg}$ ) is more effective than standard BP control (SBP target $<140 \mathrm{mmHg}$ ) to reduce the rate of CV events and mortality. Primary composite outcome was myocardial infarction and other acute coronary syndromes, heart failure, stroke and death from CV causes. A post-hoc analysis of the SPRINT confirmed that the meaning of these results can be extrapolated to patients $\geq 75$ yr-old regardless their frailty status [6]. Of note, a large number of adverse reactions (i.e. hypotension, syncope, electrolyte abnormalities, acute kidney injury or acute renal failure) were observed in the intensive treatment group, making doubtful to apply SPRINT results to clinical practice. Although the percentage of injurious falls was similar in the intensive group compared with the standard group $(7.1 \%$ vs $7.1 \%)$, the much larger occurrence of acute kidney failure, syncope, electrolyte abnormalities and hypotension (Table 1) in the intensive group [5] would underscore that a more aggressive regimen can be harmful in this clinical setting. It would be reasonably obvious that the occurrence of a $67 \%$ increase in the risk of hypotension, a $66 \%$ increase in the risk of acute renal failure and a $35 \%$ increase in the risk of electrolyte abnormalities should be avoided in elderly hypertensive patients undergoing anti-hypertensive treatment. On the other hand, a recent analysis on patient-reported outcomes among patients participating to SPRINT, showed no significant difference in terms of satisfaction with BP-care and medications, adherence to the treatment and quality of life, between patients assigned to the intensive treatment and those assigned to the standard treatment [7]. Thus, benefits of intensive BP reduction in the elderly are associated with a good patient-reported outcome, that seems to overshadow any potential side-effects. Of note, the Authors declared that when serious adverse events occurred in the intensive arm treatment, they promptly tapered medications, in that somehow altering the severity of adverse events over time.

Apart from adverse events, the amazing results of SPRINT appear of scarce reproducibility in older hypertensives in the real life. Some criticisms regarding the extrapolation of SRINT results to elderly patients have been raised: i) small sample size of patients aged $\geq 75$ (28.2\%); ii) self-BP measurement, that is consistently different from the usual approach carried out in clinical practice.

Moreover, the exclusion of patients with characteristics usually occurring in a frail old patient (e.g. diabetes mellitus, prior stroke, lack of autonomy in daily activities), limits the extrapolation of the SPRINT results to elderly patients, although exploratory analyses would encourage to apply the SPRINT results to frail patients [8]. Thus, while treatment of hypertension in older adult patients is mandatory because of not only the increased CV risk due to ageing itself, but also the high prevalence of hypertension (60-90\%) and ISH (>60\%) [9] in the elderly, the intrinsic heterogeneity of this category of patients makes difficult finding a unique BP target. Furthermore, the SBP ten- dency to decline observed in the final phase of life in patients over 80 years, irrespective of their frailty status [3], as well as the variability of on-treatment BP and outcomes depending on the grade of frailty [10], are major confounders in the assessment of benefits and risks. However, a stable BP reduction over time seems to be the best approach in the strategy of prevention of CV morbidity and mortality in older adults.

The cheering prediction of $\sim 107,500$ death prevented in United States if the results of the SPRINT would have been applied to American people [11], together with the estimated cost-effectiveness of this potential application to clinical practice, especially if the treatment would be longer than 5 years, or persisted for the patient's remaining life [12], would apparently encourage an intensive anti-hypertensive strategy in selected older hypertensives who meet SPRINT criteria. However, to date SPRINT results, albeit cheering, still remain far from being extrapolated to clinical practice, particularly in elderly patients, also because life-expectancy of these patients may be shorter than 5 years. By contrast, it is mandatory in this clinical setting to avoid serious adverse events (e.g. acute renal failure) that can dramatically worsen patient's conditions.

Furthermore, in this respect it should be taken into account that a post-hoc analysis from the Valsartan in Elderly Isolated Systolic Hypertension (VALISH) study recently showed that, an on-treatment SBP of $130-144 \mathrm{mmHg}$ in old patients with ISH was effective to reduce CV risk and mortality [9]. Nevertheless, physicians must weigh the benefits and the risks related to hypertension management in elderly patients. Thus, in agreement with ESH/ESC Guidelines [4] and the American College of Physicians statement [13], at this stage it seems better to choosing wisely a conservative SBP target of $<150 \mathrm{mmHg}$ in primary prevention. More evidence is in fact needed to underscore a positive balance between effectiveness and serious adverse events in elderly patients achieving tighter BP targets in the real life.

\section{Future perspectives}

In conclusion, physicians should be encouraged to treat hypertension in the elderly patients, even in those older than 80 years bearing in mind that cerebrovascular reactivity in hypertensive patients, being more sensitive to a sudden fall in blood pressure [14], could make easier the occurrence of lacunar stroke, especially in elderly patients [15]. However, it remains still under debate whether a more intensive BP control would be beneficial, leaving the patient free from serious adverse events. The aim of future evaluation is to assess in the real life which patients can get benefit from the SPRINT strategy. with the lowest number of adverse events [16]. At first hand, particularly frail patients should require a cautious tailored anti-hypertensive treatment, with a goal of SBP near to $150 \mathrm{mmHg}$, trying to slowly adapting the goal upon the patient's response. Tight

Table 1. SPRINT trial: percentage increase of main Serious Adverse Events in the intensive treatment group (n.1793 adverse events) as compared with the standard treatment group (n. 1736 adverse events).

\begin{tabular}{llc}
\hline Acute Kidney injury or Acute Renal Failure & $+4.1 \%$ (from $6.7 \%$ to $10.8 \%)$ & $<0.001$ \\
\hline Hypotension & $+2.3 \%$ (from $3.8 \%$ to $6.1 \%)$ & 0.001 \\
\hline Electrolyte abnormalities & $+1.8 \%$ (from $6.2 \%$ to $8.0 \%)$ & 0.02 \\
\hline Syncope & $+1.4 \%$ (from $4.6 \%$ to $6.0 \%)$ & 0.05 \\
\hline
\end{tabular}


monitoring of possible serious adverse events, and consequent prompt changes in treatment, are always advisable, with a regular assessment of frailty status [10].

\section{References}

1. Lloyd-Jones DM, Evans JC, Levy D. Hypertension in adults across the age spectrum: current outcomes and control in the community. JAMA 2005;294:466-72.

2. HYVET Study Group. Treatment of hypertension in patients 80 years of age or older. N Engl J Med 2008;358:1887-98.

3. Ravindrarajah R, Hazra NC, Hamada S, et al. Systolic blood pressure trajectory, frailty, and all-cause mortality $>80$ years of age: cohort study using electronic health records. Circulation 2017;135:2357-68.

4. Task Force Members. 2013 ESH/ESC Guidelines for the management of arterial hypertension: the Task Force for the management of arterial hypertension of the European Society of Hypertension (ESH) and of the European Society of Cardiology (ESC). J Hypertens 2013;31:1281-357.

5. SPRINT Research Group. A randomized trial of intensive versus standard blood-pressure control. N Engl J Med 2015;373:2103-16.

6. SPRINT Research Group. Intensive vs standard blood pressure control and cardiovascular disease outcomes in adults aged $\geq 75$ years: a randomized clinical trial. JAMA 2016;315:2673-82.

7. SPRINT Research Group. Effect of intensive blood-pressure treatment on patient-reported outcomes. N Engl J Med 2017;377:733-44.

8. SPRINT Study Research Group. Characterizing frailty status in the Systolic Blood Pressure Intervention Trial. J Gerontol A Biol Sci Med Sci 2016;71:649-55.

9. Yano Y, Rakugi H, Bakris GL, et al. On-treatment blood pressure and cardiovascular outcomes in older adults with isolated systolic hypertension. Hypertension 2017;69:220-7.

10. Benetos A, Bulpitt CJ, Petrovic M, et al. An expert opinion from the European Society of Hypertension-European Union Geriatric Medicine Society working group on the management of hypertension in very old, frail subjects. Hypertension 2016;67:820-5.

11. Bress AP, Kramer H, Khatib R, et al. Potential deaths averted and serious adverse events incurred from adoption of the SPRINT (Systolic Blood Pressure Intervention Trial) intensive blood pressure regimen in the United States: projections from NHANES (National Health and Nutrition Examination Survey). Circulation 2017;135:1617-28.

12. SPRINT Research Group. Cost-effectiveness of intensive versus standard blood-pressure control. N Engl J Med 2017;377:745-55

13. Clinical Guidelines Committee of the American College of Physicians and the Commission on Health of the Public and Science of the American Academy of Family Physicians. Pharmacologic treatment of hypertension in adults aged 60 years or older to higher versus lower blood pressure targets: a clinical practice guideline from the American College of Physicians and the American Academy of Family Physicians. Ann Intern Med 2017;166:430-7.

14. Malatino LS, Bellofiore S, Costa MP, et al. Cerebral blood flow velocity after hyperventilation-induced vasoconstriction in hypertensive patients. Stroke 1992;23:1728-32.

15. Buonacera A, Stancanelli B, Malatino L. Stroke and hypertension: an appraisal from pathophysiology to clinical practice. Curr Vasc Pharmacol 2017 doi: 10.2174/1570161115666171116151051 [Epub ahead of print].

16. Phillips RA. Hypertension treatment and outcomes in the era of population health, coordinated care, and medicare access and CHIP Reauthorization Act (MACRA). Circulation 2017;135:1629-31. 